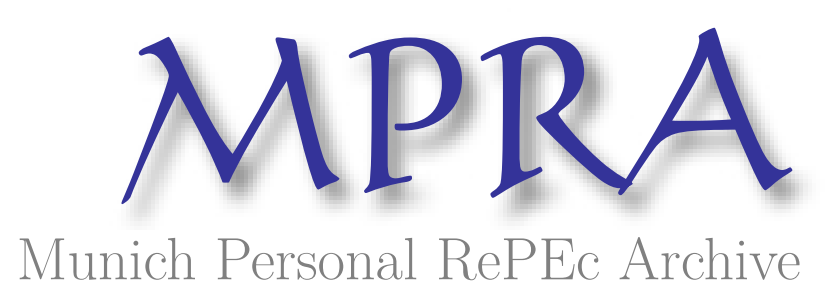

\title{
The experience curve and the market size of competitive consumer durable markets
}

Kaldasch, Joachim

EBC Hochschule Berlin

14 September 2011

Online at https://mpra.ub.uni-muenchen.de/33370/

MPRA Paper No. 33370, posted 14 Sep 2011 11:27 UTC 


\title{
The Experience Curve and the Market Size of Competitive
}

\section{Consumer Durable Markets}

\author{
Joachim Kaldasch \\ EBC Hochschule Berlin, Alexanderplatz 1, 10178 Berlin, Germany \\ (Email: joachim.kaldasch@international-business-school.de)
}

\begin{abstract}
An evolutionary model of the product life cycle is applied to derive the experience curve and the market size of (expensive) durable goods. The experience (learning) curve suggests that the real costs per unit decrease with an increasing cumulative output (Henderson's law). Based on the idea that in a competitive market firms are forced to pass cost advantages on to the price, the evolutionary model suggests that the mean price and also the mean costs are governed by an exponential decline with time. Simultaneously the mean price evolution satisfies Henderson's law. The market size is defined here by the number of active firms. The market size is shown to follow the total market revenue if the latter exhibits fast variations, else the size is nearly constant. A comparison with an empirical investigation confirms the model predictions.
\end{abstract}

Keywords: experience curve, learning curve, market evolution, evolutionary economics, economic growth, product diffusion, Gompertz diffusion, product life cycle, durable goods 


\section{Introduction}

We want to apply in this paper a previously established evolutionary model of the product life cycle to establish the experience curve and the market size of durable goods [1, 2]. The experience (learning) curve is an empirical finding that suggests that the real costs per unit decrease with an increasing cumulative output. The standard interpretation of the experience curve is that supply side advantages due to economies of scale, repetitive manufacturing and new technologies cause a decrease in the production costs $[3,4]$. The evolutionary model allows also a derivation of the market size characterized by the number of firms active in the durable market [5].

The evolutionary model of a competitive market can be understood as follows. Let us consider a durable (expensive) consumer good produced for an anonymous market. In a competitive market a number of different variants of the same good are available. These variants are called here brands. The brands are manufactured by business units, while firms may consist of a number of business units. Consumers have the choice between these brands. The key idea is that the reproduction of the brands is governed by a positive feedback mechanism. Best selling brands, will be reproduced with a much higher rate than slow sellers. In other words, the manufacturers respond on increased sales of a brand with a higher output, because they want to make profit. Further, they create variants of the best selling brands and stop the production of the slow sellers. As a result, after sufficient time, the durable good evolves such that it suits the major consumer demand.

In other words, the positive feedback mechanism is equivalent to preferential growth. Preferential growth can be formulated in terms of the Variation-Selection-Reproduction (VSR) mechanism, known form the evolution of species (for an overview [6,7]). In these terms brands (business units) play the role of species and the selective environment is given by the consumer demand. Mutations are the decisions of the manufacturers to vary the product features.

While the VSR mechanism applies to all features of the good (e.g. design, efficiency etc), derived in [1] is an evolutionary model, which focuses essentially on one feature of the good; the price. The model suggest that each brand is can be characterized by a product fitness, which is proportional to a price dependent market volume. Because the market volume increases with a decreasing price of the brand, a small price decrease increases its fitness and amplifies the unit sales (via a replicator equation). It turns out that in a competitive market the mean price is governed by an exponential decline with time. This decline, however, has the consequence that the number of potential consumers, limited by their personal income, increase. The decrease of the mean price is therefore the origin of an evolutionary diffusion process, which turns out to be governed by Gompertz equation (denoted therefore Gompertz diffusion). In order to apply the model here, we first summarize the key results relevant for an understanding of the experience curve and the size of the market.

The derivation of the experience curve is based on the idea that in a competitive market firms intend to improve their market position. This can be done by passing on cost advantages in the production of the good on to the price. If this process can be treated as causing small random price fluctuations, they are already contained in the original theory [1]. This is because the dynamics of the evolutionary (competitive) model is determined by small price fluctuations. In other words, cost fluctuations come into play in the evolution of the mean price. But if this statement is correct, the empirical finding that the cumulative output can be approximately described by a power law function of the total output, refereed to as Henderson's law, and must be regained with the evolutionary approach. This will be tested with an empirical investigation of a consumer durable. 
The number of firms can be derived from the evolutionary model on the basis of a comparison of the adaptation velocities of firms compared to the velocity of variations of the total market. If the firms cannot adapt their costs (size) sufficiently fast, compared to variations of the total market, the number of firms is governed by the total revenue that can be achieved in the durable market. On the other hand, if they can adapt sufficiently fast, we can expect that the market size is not governed by total revenue and is apart from fluctuations nearly constant.

The paper is organized as follows. In the next section the results of the evolutionary model are summarized and supplemented with a derivation of the experience curve and the market size. Then the model is applied to an empirical example, followed by a conclusion. 


\section{The Model}

We first summarize the key results of the evolutionary model derived in [1,2] where the product life cycle of durable goods is derived explicitly. However, because it is not relevant for the consideration of the experience curve, we want to confine here to expensive consumer durables and neglect the initial Bass diffusion.

The demand side is characterized in this model by an ensemble of agents who are interested in purchasing the consumer durable, while the total number of agents is denoted as the market potential $M$. The personal income distribution can be divided into a lower class contribution (lognormal/exponential distribution) and an upper class (Pareto distribution) [8$10]$. We assume that, in difference to luxurious goods, the upper class can always afford the good, indicated by the contribution $M_{U}$. The market volume, $V(\mu)$ has an additional contribution from the lower class. It is determined by those consumers, who can afford the good for a real price:

$\mu=\frac{p}{I_{L}}$

while $I_{L}$ is the mean income of the lower class and $p$ is the nominal price. We further assume that there exists a natural (minimum) price $\mu_{m}$, at which the market volume is equal to the market potential:

$V\left(\mu_{m}\right)=M$

(2)

For $\mu>\mu_{m}$, the market volume $V(\mu)$ can than be written as the sum of a contribution from the upper class $M_{U}$, which also includes industrial agents, and a price dependent part from the lower class of the form:

$$
V(\mu) \cong M_{L} \exp \left(-\frac{\left(\mu-\mu_{m}\right)^{2}}{2 \Theta^{2}}\right)+M_{U}
$$

where $\Theta$ is a constant specific for the durable market and:

$$
M=M_{L}+M_{U}
$$

Because the model is developed in terms of continuous variables, we establish densities by scaling extensive variables with the market potential $M$. The density of the market volume becomes:

$$
v(\mu)=\frac{V(\mu)}{M}
$$

with $0 \leq v(\mu) \leq 1$. 
The supply side of the market is characterized by a number of different variants of the durable good, denoted as brands (models) having similar utility properties. We assume that each brand is manufactured by a business unit, indicated with index, $i$, while $N$ is the total number. The absolute number of products of the $i$-th brand sold per unit time is denoted $Y_{i}$, and the corresponding density is:

$$
y_{i}=\frac{Y_{i}}{M}
$$

with total unit sales:

$$
y_{t}=\sum_{i=1}^{N} y_{i}
$$

The mean price is defined as:

$$
\langle\mu(t)\rangle=\frac{1}{y_{t}(t)} \sum_{i=1}^{N} y_{i}(t) \mu_{i}(t)
$$

where single brackets indicate the average over the unit sales. A key result of the evolutionary approach is that due to the mutual competition between the brands the mean price evolves according to:

$$
\langle\mu(t)\rangle=\mu_{0} e^{-a t}+\mu_{m}
$$

where $\mu_{0}$ is the price at $t=0$, and the constant parameter $a$ is denoted as price decline rate. The price decline rate has the form, $a \sim \operatorname{Var}\left(P_{\mu}\right)$, where the variance of the price distribution is:

$\operatorname{Var}\left(P_{\mu}\right)=\left\langle\mu_{i}^{2}-\langle\mu\rangle\right\rangle$

Note that $a$ is zero for a monopoly market, because the variance vanishes. Monopolies are therefore not governed by the evolutionary model and have to be excluded from our considerations!

The evolutionary model suggests that the product life cycle (PLC) of consumer durables can be described by the total unit sales:

$y_{t}(t)=y_{t f}(t)+y_{t r}(t)$

where $y_{t f}(t)$ is the total first purchase and $y_{t r}(t)$ is the total repurchase of the good.

We want to confine here to the case that $M_{U}<<M_{L}$ in order to neglect the initial Bass diffusion (expensive durable) and set $M_{U} \approx 0$. The total first purchase is than determined by the expansion of the market volume due to the decrease of the mean price. That means, new 
adopters are created by the increase of the market volume. The corresponding first purchase sales can be obtained from:

$$
y_{t f}(t)=\frac{d n(t)}{d t}=\frac{d v(\langle\mu(t)\rangle)}{d t}
$$

where $n(t)$ is the adopter density and the market volume is given by Eq.(3). Inserting Eq.(9) in Eq.(3), the time derivative can be performed and we obtain for the first purchase unit sales:

$y_{t f}(t)=2 a k n(t) \exp (-2 a t)$

with the adopter density:

$n(t)=n_{0} \exp \left(-k e^{-2 a t}\right)$

while

$k=\left(\frac{\mu_{0}}{2 \Theta^{2}}\right)^{2}$

and $n_{0} \approx 1$. The evolutionary model suggests therefore that the diffusion process caused by the expansion of the market volume is determined by Gompertz diffusion.

The repurchase sales are considered as the sum of total replacement $y_{t R}$ and total multiple purchase $y_{t m}$ such that :

$y_{t r}(t)=y_{t m}(t)+y_{t R}(t)$

Replacement purchase is determined by the probability distribution, $\Gamma(t)$ of product failure over the population of units. The model assigns replacement purchase to those purchase events associated with the mean product life time $t_{p}$. Replacement purchase can be approximated for $t \geq t_{p}$ by:

$y_{t R}(t) \cong r y_{t f}\left(t-t_{p}\right)$

else, $y_{t R}{ }^{B}(t)=0$, while $r>0$ is the fraction of previous sales suffered form replacement purchase. Replacement purchase induces therefore periodic variations of the unit sales with a periodicity given by the average product lifetime $t_{p}$. The standard theory of economic fluctuations variations due to repurchase are known as Juglar cycles [11]. Any other repurchase decision, not correlated with the first purchase fundamental wave, is denoted here as multiple purchase. Since, multiple purchase must be proportional to the actual number of adopters, $n(t)$, the unit sales can be approximated by: 
$y_{t m}(t)=q n(t)$

(18)

where $q>0$ is a multiple purchase rate. Note that for very cheap consumer durables (and for non-durables), the parameter $q$ can be considered to be also a function of the price. Here this parameter is treated as a price independent constant.

\section{The experience curve}

The experience curve is the empirical finding that the real costs per unit $c_{i}(t)$ decrease during the PLC. The cost per unit of the $i$-th model is defined as:

$c_{i}(t)=\mu_{i}(t)-g_{i}(t)$

where $g_{i}(t)$ is the profit per unit (markup). Both $c_{i}(t)$ and $g_{i}(t)$ are real variables, i.e. scaled by $I_{L}$. Performing the average in Eq.(19) we get for the mean costs per unit:

$$
\frac{\langle c(t)\rangle}{\langle\mu(t)\rangle}=1-\langle\alpha(t)\rangle
$$

where $\alpha=g / \mu$ is a real profit margin. For (expensive) durable goods the profit margin of the surviving business units is usually of the order $0<\alpha(t)<1$. The profit margin can be written as the sum of a time independent contribution $\alpha$ and time dependent fluctuations $\delta \alpha(t)$ : $\alpha(t)=\alpha+\delta \alpha(t)$. In order to survive the time independent contribution of a brand must be large compared to the fluctuations $\alpha>\delta \alpha(t)$. Because $\alpha$ is small, the fluctuations of the profit margin must be even smaller. Neglecting the contribution of small fluctuations in Eq.(20) we obtain approximately:

$$
\frac{\langle c(t)\rangle}{\langle\mu(t)\rangle} \cong 1-\alpha=\text { const }
$$

That the profit margin in durable market can be considered to be nearly constant was also found in empirical investigations [12].

The competitive model derived in [1,2] implicitly assumes that business units pass their competitive advantage on to the price of the brand. Treating these small price variations as random independent events the evolutionary model predicts that the mean price decreases exponentially according to Eq.(9). With Eq.(21) we obtain therefore that, up to a constant, the evolution of the costs per unit follow the mean price, $\langle c(t)\rangle \sim\langle\mu(t)\rangle$.

The time dependent decrease of the real costs per unit is usually displayed as a result of a learning process in the form of a power law (Henderson's law) [13]:

$\langle c(t)\rangle \sim x(t)^{-\beta}$ 
where $x(t)$ is the cumulative volume of production and $\beta$ is the elasticity of costs with regard to output. Empirical investigations suggest a decline of the costs per unit of the order of 10$25 \%$ for a doubling of the cumulative output. Because the mean costs follow the mean price, the model suggests that the mean price is governed on the one hand by Eq.(9) and on the other by Henderson's law Eq.(21).

\section{The market size}

We want to characterize the market size by the number of firms $N^{\prime}(t)$. The market size is confined by the condition that the total costs cannot be larger than the total revenue of the durable market. The total revenue is given by:

$R(t)=\langle\mu(t)\rangle y_{t}(t)$

and the total costs are:

$$
C(t)=\langle c(t)\rangle y_{t}(t)
$$

With these relations Eq.(21) can be rewritten as:

$$
\frac{C(t)}{R(t)} \cong 1-\alpha
$$

Because $\alpha>0$ the condition of a limited market size $(C(t)<R(t))$ is fulfilled. We further introduce the mean costs per firm defined as:

$$
\langle\langle C(t)\rangle\rangle=\frac{1}{N^{\prime}(t)} \sum_{j=1}^{N^{\prime}} C_{j}(t)=\frac{C(t)}{N^{\prime}(t)}
$$

where the double brackets indicate the average over the number of firms. Using Eq. (25) and taking the time derivative we obtain:

$$
\frac{d C(t)}{d t}=N^{\prime}(t) \frac{d\langle\langle C(t)\rangle\rangle}{d t}+\langle\langle C(t)\rangle\rangle \frac{d N^{\prime}(t)}{d t}=(1-\alpha) \frac{d R(t)}{d t}
$$

The key idea to derive the market size is the empirical fact that the production costs cannot adapt arbitrarily fast to demand variations (due to fixed costs). In the growth phase of the PLC the total revenue undergoes fast variations. The revenue growths due to increasing unit sales but decrease with the mean price. In this phase of the PLC some manufacturers cannot adapt their size sufficiently fast to the market evolution. Therefore we suggest that the revenue varies much faster than the mean costs: 
$\frac{d R}{d t}>>\frac{d\langle\langle C(t)\rangle\rangle}{d t}$

The evolution of the number of firms can be given approximately by the integration of Eq.(27), while condition Eq.(28) can be satisfied by setting $d\langle\langle C(t)\rangle\rangle / d t \approx 0$. Therefore, the number of business units is governed in this period by the total revenue of the market:

$N(t) \approx \frac{(1-\alpha)}{\langle\langle C(t)\rangle\rangle} R(t) \sim b\langle\mu(t)\rangle y_{t}(t)$

The number of firm's first increase and then decrease following on average $R(t)$. The period of a decreasing market size is known as shakeout [5]. Because firms intend to grow, they slowly decrease the costs and may go bankrupt when the total revenue of the market decreases faster. This period is accompanied with an increased unemployment. It has its origin according to the presented theory in the competition between the brands, contained in the mean price evolution.

In the maturity phase of the PLC, on the other hand, the total revenue varies slowly such that:

$\frac{d R}{d t} \sim \frac{d\langle\langle C(t)\rangle\rangle}{d t}$

(30)

The firms can adapt their costs sufficiently fast, respectively can compensate fluctuations due to a diversified product portfolio. Hence the market size $N^{\prime}(t)$ is approximately constant in this phase of the PLC.

\section{Comparison with Empirical Results}

Note that the PLC derived in this model characterizes the main trend of the unit sales of a durable market. Not contained are deviations due to events (e.g. the introduction of a new brand [2]). The aim of the present model is therefore not to make precise forecasts, but to understand the key relationships that drive the evolution of a durable market. The model suggests: 
I) A market of expensive durables is governed by Gompertz diffusion and is related to an exponential decline of the mean price. The PLC suffers from periodic variations of the unit sales caused by the finite lifetime of the good.

II) The mean costs per unit are governed by the evolution of the mean price. Because the mean price is determined by an exponential decline, also the costs per unit will exhibit this relationship. Displaying the mean price as a function of the cumulative total output, however, Henderson's law must be regained.

III) The number of firms is governed by the total income in periods of fast variations of the total market revenue. Otherwise the market size should be nearly constant.

We want to compare the predictions of the model with empirical data available for the evolution of the US market of Black \& White (B\&W) TV sets as summarized by Wang [5].

Displayed in Fig.1 are the nominal price (triangle) and the market penetration (circles) of B\&W TV's. The fat lines are applications of the present model with the parameters given in $[1,2]$. As can be seen the mean price decreases according to an exponential law, while the market penetration can be described by Gompertz diffusion. Displayed in Fig.2 are the corresponding unit sales, which are the result of first and repurchase processes. First purchase is a combination of Gompertz and of Bass diffusion not further specified here (see [1,2].)

Displayed in Fig. 3 is this empirical mean price as given in Fig.1 as a function of the empirical cumulative output obtained from Fig.2. Also shown is a learning curve of the form Eq.(22) with $\beta=-0.32$, which expresses a $20 \%$ reduction of the mean price for every doubling of the cumulative output. As can be seen the empirical data of the mean price can be approximately described by Henderson's law. But it is also given by an exponential decline as shown in Fig.1. This result suggests that the mean price dynamics is governed by the competition between the brands, implicitly containing decreasing production costs.

Finally displayed in Fig.4 is the empirical number of firms in this durable market [5]. Eq.(29) suggests that the market size is essentially determined by the revenue that can be achieved, as long as the total unit sales exhibit considerable variations with time. The fat line in this figure represents the empirical unit sales multiplied with the mean price and a factor $b \approx 44$ per US\$ (Eq.(29)). Up to the middle of the nineteen sixties the number of firms is roughly proportional to the market revenue. After this period the number of firms approaches a nearly constant value. This trend is according to the model a consequence of the adaptation velocities of the firms to demand fluctuations.

\section{Conclusion}

The evolution of a market of (expensive) durable goods can be visualised as schematically displayed in Fig.(5), neglecting the initial Bass diffusion [1]. Shown is the market volume as a function of the real price, $v(\mu)$. It has a maximum at the natural price $\mu_{m}$. The market volume represents an effective demand curve of the durable good. The price distribution $P_{\mu}$ contains the mutual competition between the brands and is derived explicitly in [1]. The theory suggests that the mean price $\langle\mu(t)\rangle$ approaches $\mu_{m}$ asymptotically governed by an exponential law (insert, $\mu(t))$. At the same time the market volume expands associated with a diffusion process governed by Gompertz equation (insert, $n(t))$. Displayed in the other insert is the product life cycle, characterized by the total unit sales $y_{t}(t)$ (fat line). It is the sum of first purchase $y_{t f}(t)$, multiple purchase $y_{t m}(t)$ and replacement purchase $y_{t R}(t)$ (dotted lines). The last contribution induces periodic variations of the unit sales determined by the mean lifetime of order 8-12 years (Juglar cycles).

The market size, given by the number of firms $N(t)$, is limited by the total revenue $<\mu(t)>y_{t}(t)$ (dotted line). The shakeout has its origin in the presented model in the limited 
ability of firms to adapt to variations of the total revenue. When the total revenue varies slowly, the number of firms is nearly constant (except for rapid market variations caused for example by dramatic events). Firms can vanish form the market caused by bad management or replacement of their products and also new firm may enter the market. These contributions can be considered to be small compared to the evolution of the total market revenue. The comparison with empirical data suggests that the model covers the main trend.

The mutual competition forces firms to pass their cost advantages on to the price. As long as these advantages can be treated as small random steps, the cost evolution is contained in the evolution of the mean price. Therefore the mean price evolution displayed as a function of the cumulative output reflects Henderson's law. But is also governed by the evolutionary dynamics of the competitive market and exhibits therefore an exponential decline with time. Both effects are found in the empirical data considered here.

Wene specified two phases of the learning process [14]. The first phase is dominated by public R\&D support and a second phase dominated by learning investments on the market. Obviously the presented model describes the latter phase. In terms of the evolutionary VSR mechanism, cost advantages lead to a preferential growth of the brand via a better price in the selective environment formed by the consumer price expectations. Hence the business units (firms) are under permanent stress to decrease their costs with learning investments in particular in during shakeout. On the other hand if they are subsidised, the price decline and therefore cost reduction is delayed [15]. Finally we have to emphasize that the model is not applicable to a monopoly market. 


\section{References}

[1] J. Kaldasch, Evolutionary Model of an Anonymous Consumer Durable Market, Physica A, 390, (2011) 2692-2715.

[2] J. Kaldasch, The Product Life Cycle of Durable Goods, Preprint condmat/11090828 (2011).

[3] T.P. Wright, Factors Affecting the Cost of Airplanes, Journal of Aeronautical Sciences, 3(4) (1936) 122-128.

[4] S. Hollensen, Marketing Management: A Relationship Approach, Pearson Education (2010).

[5] Z. Wang, Income Distribution, Market Size and the Evolution of Industry, Review of Economic Dynamics, 11(3), 542-565, (2007).

[6] R. Feistel, W. Ebeling, Physics of Self-Organization and Evolution, Wiley VCH (2011).

[7] E.D. Beinhocker, The Origin of Wealth, Harvard Business School Press (2006).

[8] C. A. Silva, V. M. Yakovenko, Temporal evolution of the "thermal" and "superthermal" income classes in the USA during 1983-2001, Europhys. Lett. 69 (2005) 304-310.

[9] V.M. Yakovenko, Econophysics, Statistical Approach to, in Encyclopaedia of Complexity and System Science, edited by R. A. Meyers, Springer (2009).

[10] V.M. Yakovenko, J. B. Rosser, Colloquium: Statistical Mechanics of Money, Wealth, and Income, Reviews of Modern Physics 81, (2009) 1703-1725.

[11] L. Tvede, Business Cycles, History, Theory and Investment Reality, Jon Wiley \& Sons (2006).

[12] W.L. Hieber, Lern- und Erfahrungskurveneffekte, Vahlen, München (1991).

[13] R. M. Grant, Contemporary strategy analysis Blackwell Pub. (2004).

[14] C.-O. Wene, Stimulating Learning Investments for Renewable Energy Technology, EMF/IEA/IEW Workshop, Stanford (2000).

[15] P. Söderholm, G. Klassen, Wind Power in Europe: A Simultaneous Innovation-Diffusion Model, Environmental and Resource Economics 36 (2007) 163-190. 
Figures

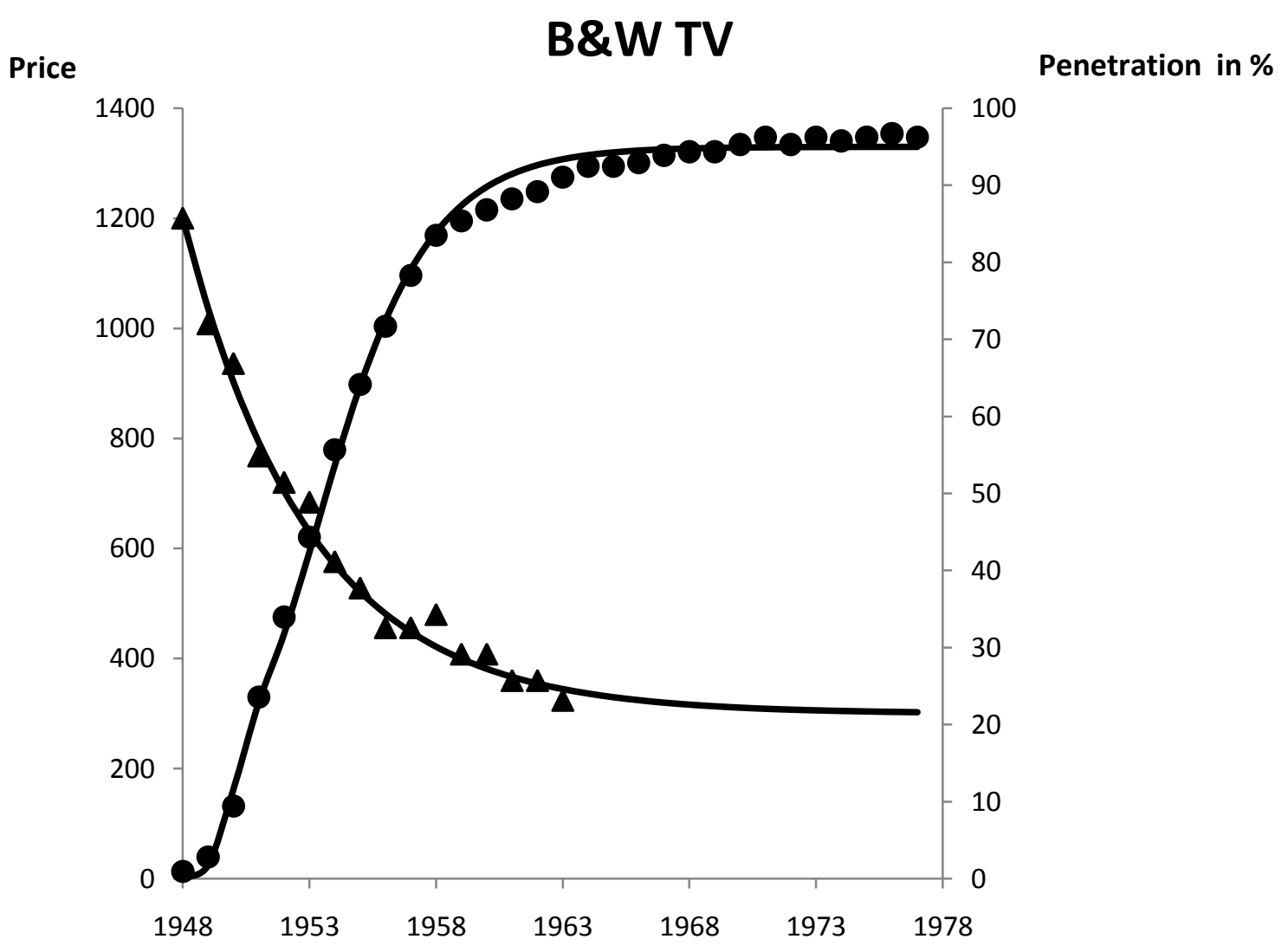

Figure 1: Evolution of the price in US \$ (triangles) and the percentage market penetration (circles) of Black \& White TV sets in the USA [1,2]. 
Sales $\left({ }^{*} 10^{6}\right)$

\section{B\&W TV}

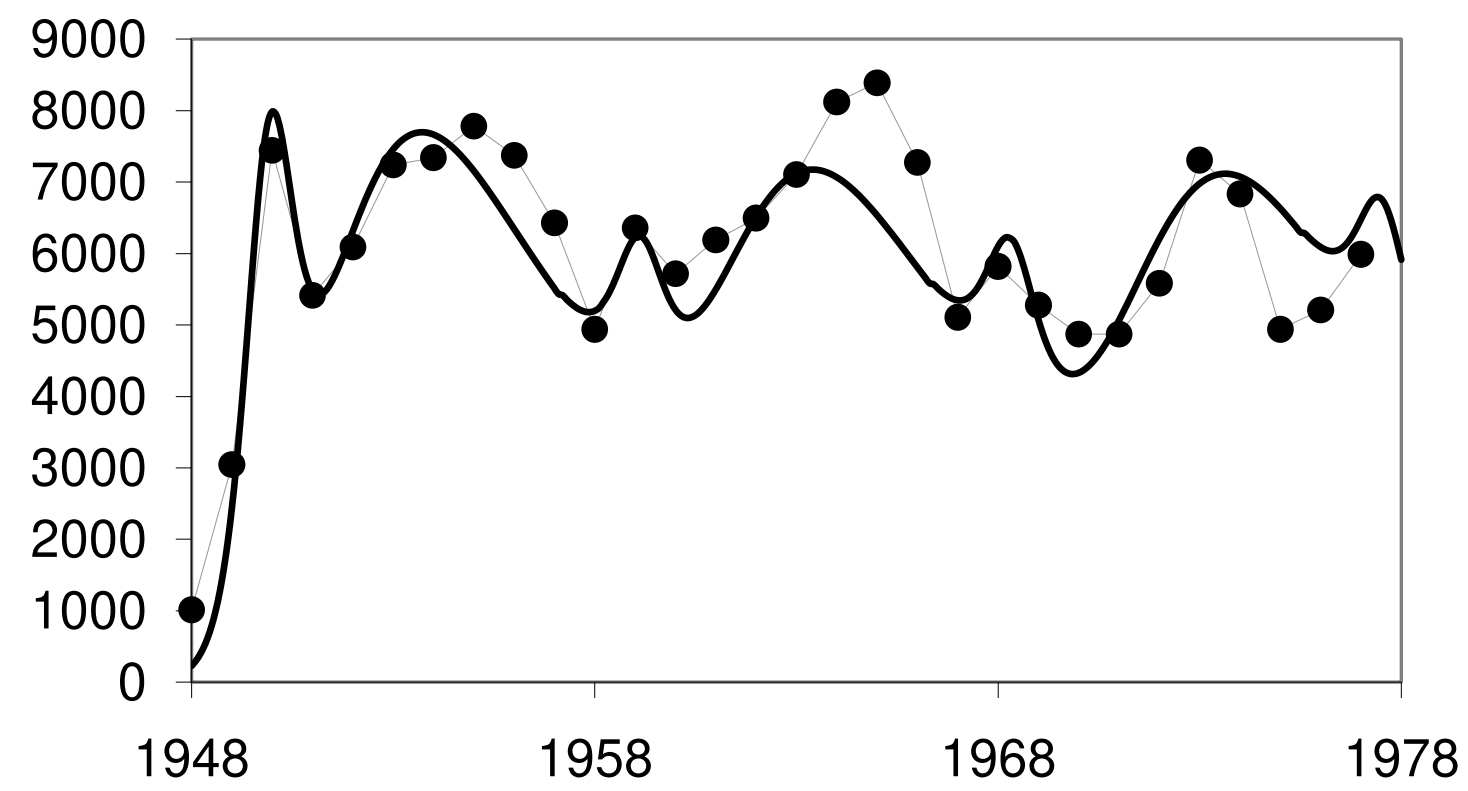

Figure 2: Evolution of the unit sales of Black \& White TV sets in the USA [1,2]. The fat line is a fit of the product life cycle. 


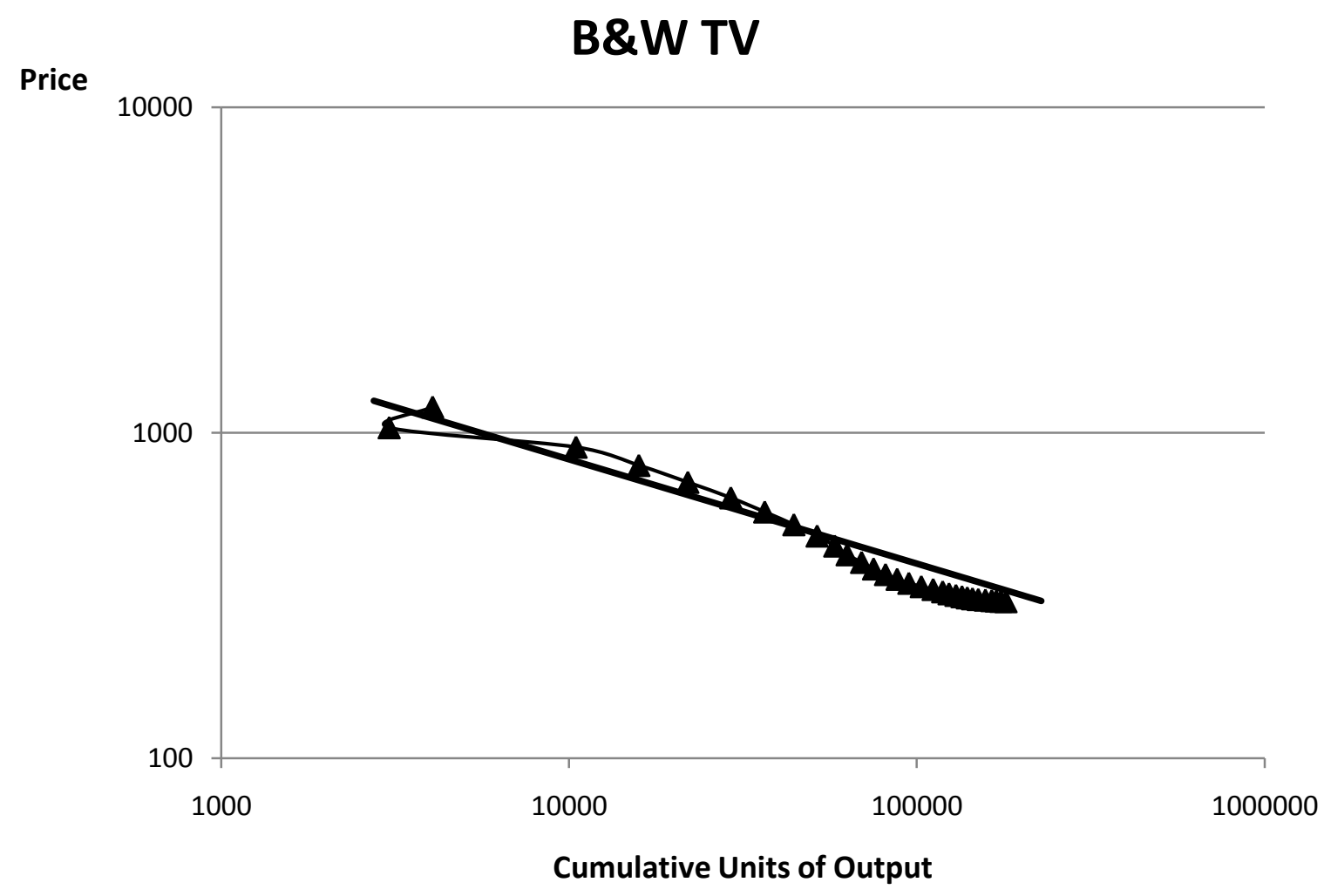

Figure 3: Experience curve of B\&W TV sets in the USA. The line expresses Henderson's law with $20 \%$ reduction of the price in US \$ for every doubling of the cumulative output. 


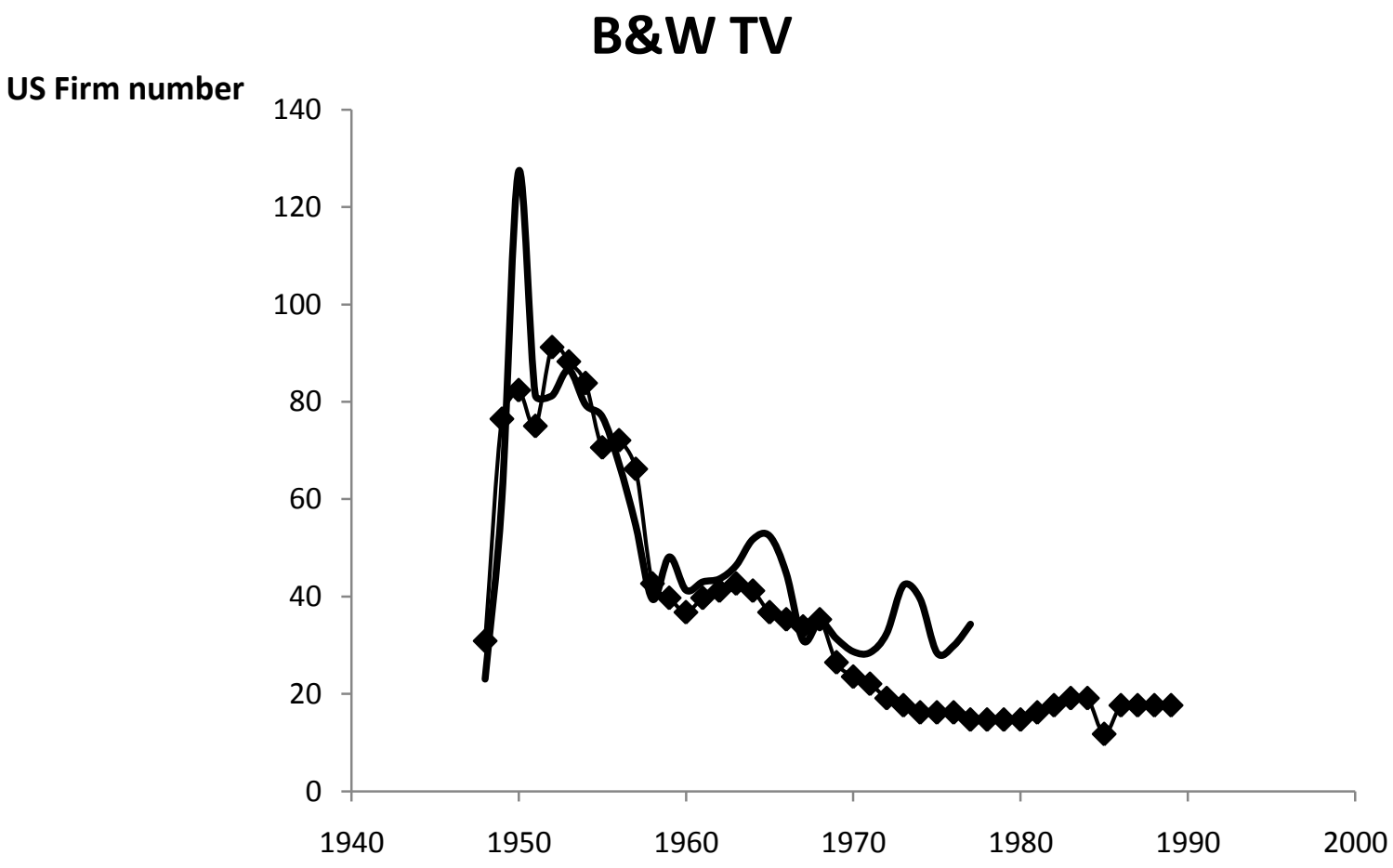

Figure 4: The market size of US Black \& White TV sets [5]. The fat line represents the total revenue of the durable market. 


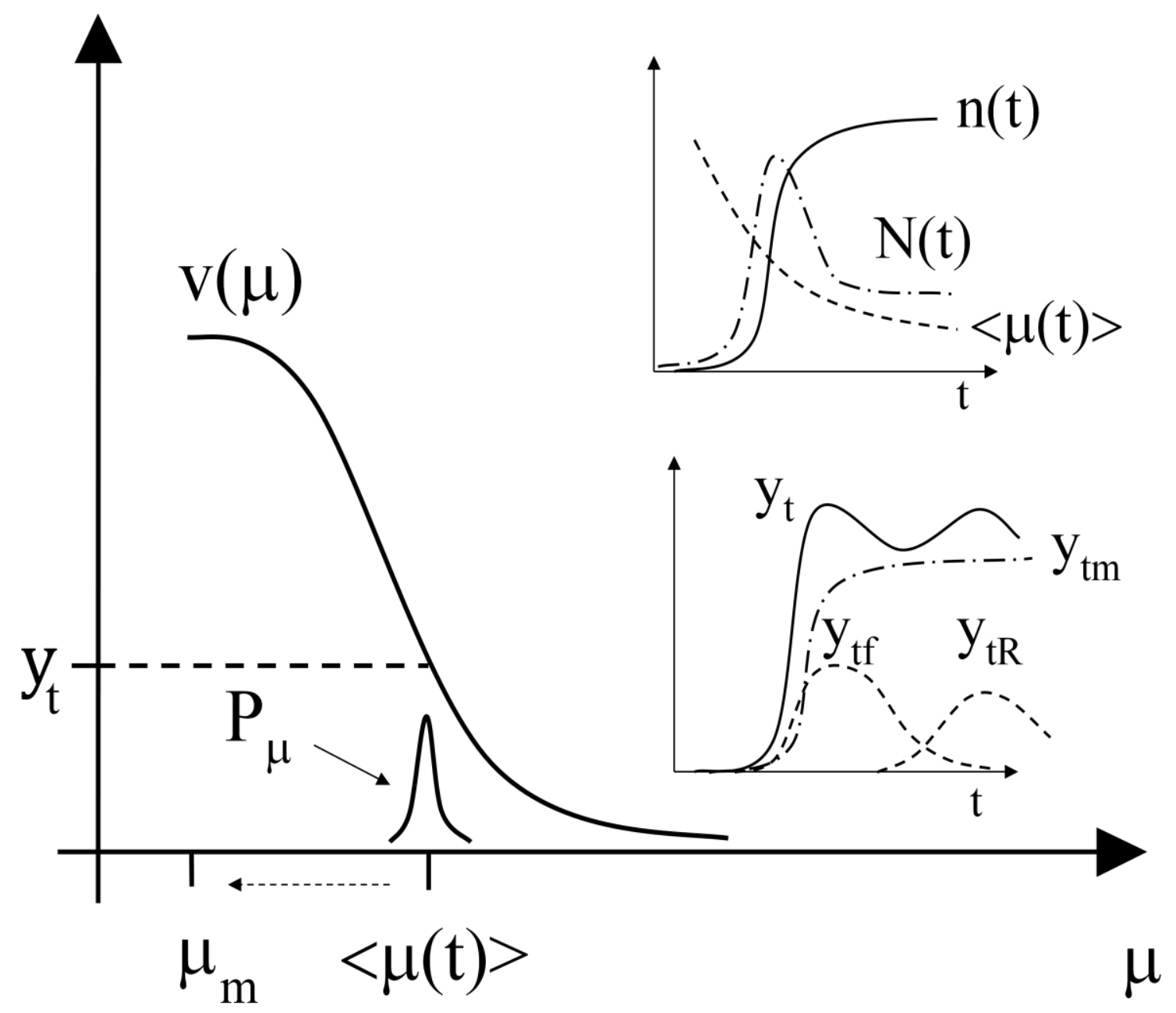

Figure 5: Schematically displayed is the evolution of a durable market [1]. 